\title{
Codificação Adaptada à Modulação em Sistemas Rádio Digitais
}

\author{
Karl J. Friederichs e R.M. Dauscha
}

Este artigo apresenta um método de codificação para correçāo de erro do tipo FEC (Forward Error Correction) com uma eficiente redundância para sistemas rádio digitais e analisa sua implementação e desempenho. Ganhos de codificação de até $6 \mathrm{~dB}$ podem ser alcançados aplicando-se códigos do tipo CSOC (Convolutional Self Orthogonal Code). Para alcançar a requerida redundância, necessita-se apenas de um pequeno aumento da taxa de transmissão. Mesmo em sistemas com grau de modulação elevado, como por exemplo 256 QAM, a implementaçáo do decodificador resulta num moderado custo de hardware.

\section{Introdução}

Sistemas rádio digitais fazem uso de modulaçáo em quadratura e amplitude de grau $M$ ( $M-Q A M$, onde $M=4,16,64$ e 256), visando atingir uma alta eficiência na largura de faixa de transmissáo disponível [1]-[3].

Os sistemas 64 e 256 QAM, devido ao grande número de sinais distintos, necessitam de uma razão sinal/ruído muito mais alta, para uma trasmissāo confiável dos dados, do que os sistemas 4 e 16 QAM. Além disso, a modulaçāo QAM de grau elevado é muito mais suscetível a imperfeiçóes sistêmicas, como distorçōes náo lineares ou recuperação nāo ideal da portadora.

A vulnerabilidade de sistemas rádio digitais com uma eficiente largura de faixa pode em grande parte ser reduzida utilizando-se uma codificaçāo para correçāo de erro [4]. Tolera-se, porém, em muitas aplicaçōes, apenas um pequeno acréscimo percentual na taxa de transmissão, visando a inclusão de redundância.

K.J. Friederichs é engenheiro da Siemens A.G., Transmission Systems.

R.M. Dauscha é engenheiro da Equitel S.A., Desemvolvimento - Transmissão. 
Para uma utilização ericiente dessa redundância, deve-se ter como objetivo um bom cesamento entre codificaçāo e modulaçāo. A aplicaçāo clássica de códigos em blocos, onde a correção de erro é baseada simplesmente em propriedades algébricas e näo em informaçāo sobre os bits de decisāo suave ou sobra o formato da modulação, é ineficiente. Um método eficaz é a modulaçäo tipo TCM ("Trellis Coded Modulation") de Ungerboeck, na qual a redundância é transmitida com um alfabeto ampliado do sinal e onde se utiliza uma decodificação do tipo Viterbi [5] e [6]. Porém, a implementação prática de um rádio digital TCM transmitindo 8 bits de dados por janela de tempo ("ime slot") com uma taxa de $30 \mathrm{MHz}$ resulta em um custo de hardware muito grande, mesmo utilizando-se modernas tecnologias VLSI ("Very Large Scale integration"i"). Adicionalmente, nessas aplicaçōes o desempenho teórico esperado do TCM é degradado devido a imperfeiçōes sistêmicas, como por axemplo, un desvio de fase da portadora [7]. Em outro estudo [8], Mecklenburg et al descrevem um esquema de correçáo de erro com uma eficiente redundêncie a olicávei a sistemas QAM, onde os bits de dados sāo mapeados nos símbolos a serem transmitidos de acordo com o código Gray. Neste presente artigo, utiliza-se o mapeamento por partição de conjuntos introduzicio por Ungerboeck [G] para a constelação convencional de 256 QA.M. O resutiado é um esquema de codificação com um reduzido acréscimo na taxa de transmissäo. A esse tipo de modulaçāo deu-se o nome de UC-QAM "Underlying Coded" - QAM). Ao final deste artigo säo apresentados resultados de cálculos e simulaçóes de desempenho de alguns códigos.

\section{UC - QAM}

Na tramsmissäo de sinais comi modulaçāo multi-nível, corrompida por ruído gatssiano, a probabilidade de erro de símbolo decresce exponencialmente com o acréscimo dá distância Euclidiana entre os símbolos. Desta forma, erros de transmissão mais prováveis sāo os chamados erros de passo simples, ou seja, falsificaçōes em símbolos adjacentes, enquanto erros de dois ou mais passos ocorrem com menor probabilidade. Entáo, uma codificeça de erro eficiente deve levar em conta a probabilidade de todos os tipos possiveis de erro [9]. Seria um desperdício de redundância tentar corrigir todos os tipos de erros com o mesmo esforço. Pode-se afirmar que é interessante concentrar os esforços na correçāo de erros de um passo, em cada direçāo do plano complexo. 
A seguir, consideramos um sistema 256 QAM que transmite 8 bits por "time slot". Como só se deseja corrigir erros de um passo, nāo se faz necessário codificar todos os bits, mas sim, apenas o bit menos significativo do símbolo transmitido no canal em fase (I) ou em quadratura (Q). A Fig. 1 mostra um diagrama em blocos do codificador. Inicialmente, o feixe de dados serial é paralelizado e a taxa de transmissāo de símbolos é aumentada de tal forma a criar espaço livre para a inserçáo de redundância na seqüência de bits menos significativos nos canais I e Q. Os dois codificadores, um para cada canal, sāo inseridos apenas para os bits menos significativos e geram bits de verificaçāo multiplexados nos momentos corretos da seqüência. A seguir, um modulador de quadratura realiza o mapeamento de 256 QAM. Desta maneira, os dois bits codificados entre os 8 bits dividem a constelaçāo 256 QAM em quatro sub-campos com uma distância mínima aumentada, enquanto os bits nāo codificados determinam a posiçāo do sinal no sub-campo. Uma descrição detalhada sobre essa divisão encontra-se em [6]. Qualquer erro de um passo ocasiona uma mudança de sub-campo que pode ser detectada pelo decodificador $\cup C$, a depender da capacidade de detecçáo e/ou correçāo do código utilizado.

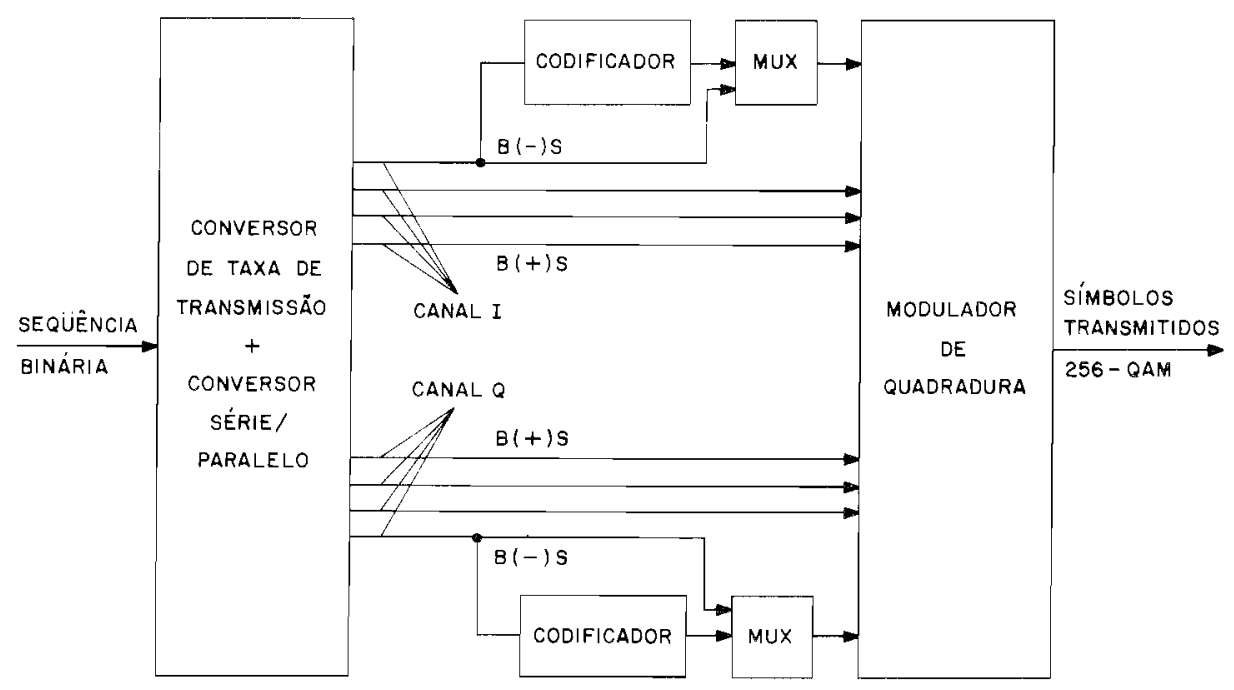

Figura 1. Diagrama em blocos do codificador UC. 
Erros de dois passos sāo ignorados, uma vez que estes nāo provocam mudança de sub-campo. Neste caso, pode-se mostrar que o máximo ganho atingivel está limitado em $6 \mathrm{~dB}$. Ganhos maiores podem ser conseguidos codificando mais que dois bits por símbolo. Em suma, o código tipo UC protege apenas os símbolos pertencentes a um sub-campo.

Um aspecto bastante interessante do esquema de codificaçāo descrito é que a taxa de codificação global $R_{g}$ é sensivelmente menor que a taxa $R_{c}$ do código UC. Genericamente, para sistemas M-QAM com $M 2^{2 m}$, $m=1,2, \ldots$ é válida a seguinte relação:

$$
\left(1-R_{c}\right) /\left(1-R_{g}\right)=\log _{2}(M)^{1 / 2}
$$

Para ilustrar essa propriedade de compressão, pode-se constatar na Fig. 2 uma seqüência de dados do canal Q(256-QAM). Nesse exemplo, a taxa global é $R_{g}=35 / 36$ enquanto o código apresenta uma taxa $R_{c}=8 / 9$. Ou seja, uma baixa redudância sistêmica de $2,86 \%$ transforma-se numa redundância de código de $12,5 \%$. A perda por aumento de faixa nesse exemplo perfaz $0,1 \mathrm{~dB}$.

CANAL Q

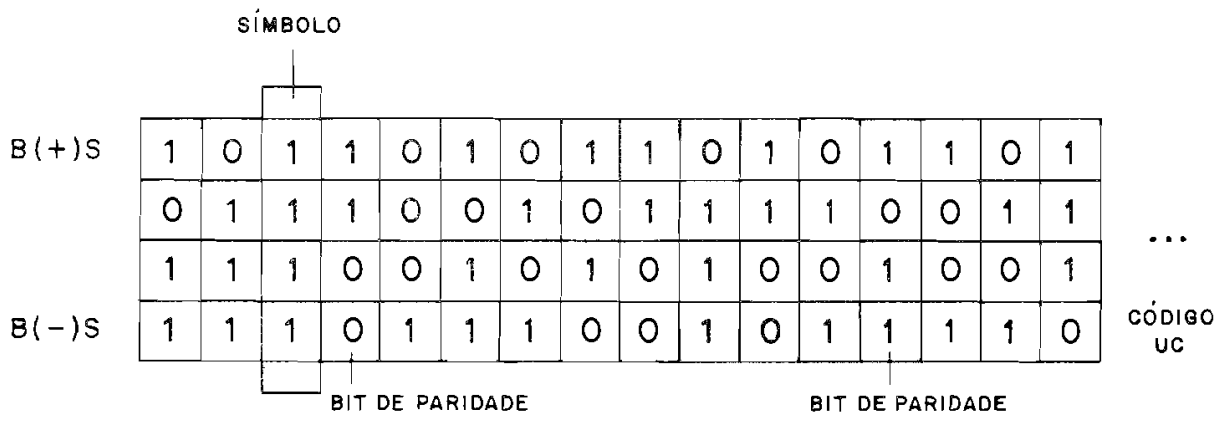

$$
\begin{array}{lll}
\text { TAXA CÓDIGO } & R_{C}=8 / 9 ; & \text { REDUNDÂNCIA DO CÓDIGO }=12,5 \% \\
\text { TAXA GLOBAL } & R_{g}=35 / 36 ; & \text { REDUNDÂNCIA SISTÊMICA }=2,86 \% \\
\text { GENERICAMENTE } & \frac{1-R C}{1-R g}=\log _{2} \sqrt{M} & M=\text { NÚMERO DE SÍMBOLOS }
\end{array}
$$

Figura 2. Princípio geral da codificaçāo UC. 
O diagrama em blocos do decodificador é mostrado na Fig. 3. Como no codificador, existem dois ramos iguais, um para cada canal I e $Q$. Vamos considerar por simplicidade, apenas um ramo. Inicialmente, os símbolos recebidos são quantizados com uma resolução de três ou quatro bits de decisão suave. Os bits de decisão abrupta e o bit mais significativo dos bits suaves sáo atrasados de acordo com o tempo de processamento do decodificador e são aplicados à unidade de correçāo. Conforme o conceito de codificação UC, apenas o bit menos significativo dos bits de decisão abrupta é aplicado ao decodificador.

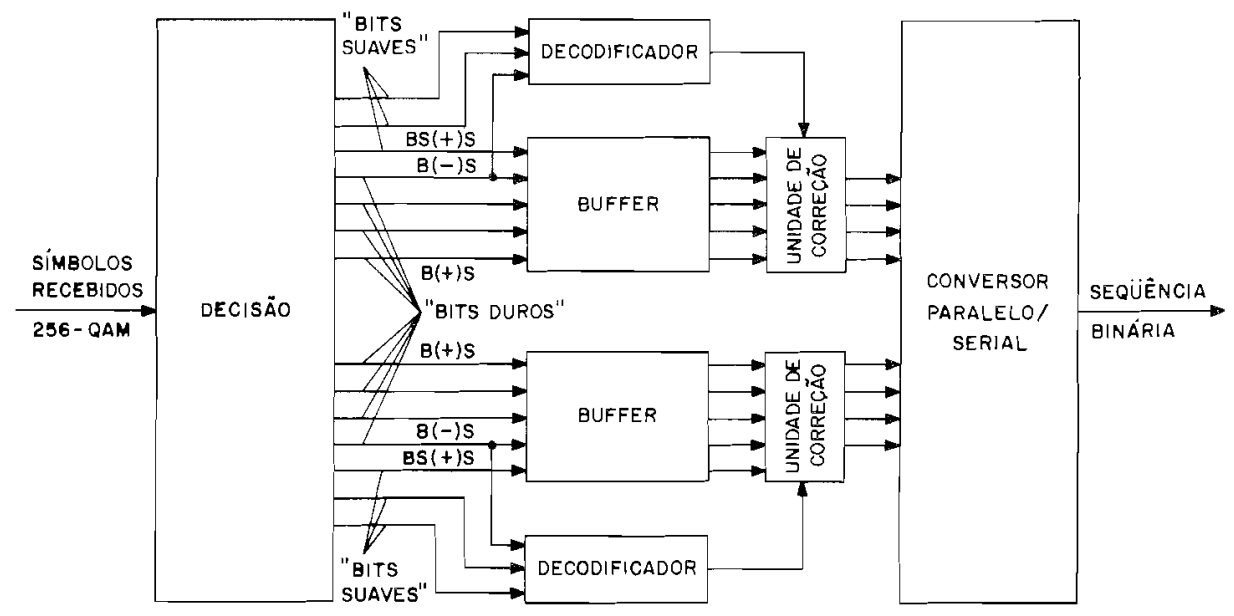

Figura 3. Diagrama em blocos do decodificador UC.

Além disso, o decodificador é alimentado com bits de decisāo suave, contendo informaçāo sobre a distância do símbolo recebido em relaçāo ao limiar mais próximo. Caso o decodificador detecte um erro, a unidade de correção é ativada no instante em que o símbolo correspondente aparecer na saída do buffer. Dependendo do bit mais significativo da decisāo suave a unidade de correção decide se o nível do símbolo supostamente errado deva ser 
elevado ou decrescido de um passo. Ao final, os símbolos corrigidos sāo convertidos num feixe serial.

$\mathrm{Na}$ estrutura adotada, foram tratados dois ramos de codificaçāo separados, um para cada canal, | e Q. A princípio, isso não é necessário porque a seqüência dos dois bits menos significativos pode ser processada conjuntamente, usando apenas um codificador e um decodificador. Razóes para esse tratamento em separado dos canais le $Q$ sáo a velocidade de processamento mais lenta e a descorrelação que se obtém caso as amostras ruidosas dos canais I e $Q$ sejam correlacionadas (distorçōes náo-lineares).

\section{Cálculo de Desempenho e Resultados de Simulação}

A princípio, qualquer código corretor de erro e algoritmo decodificador pode ser usado em conjunto com o UC-QAM. Visando obter o meihor casamento possível com as características do canal, é necessário que o algoritmo decodificador possa utilizar informaçōes de decisão suave. Critérios adicionais para a escolha de codificação UC são a taxa de codificaçāo $R_{c}$, a complexidade do decodificador e a velocidade de processamento.

Se a redundância do sistema é limitada a valores extremamente pequenos (até $3 \%$ ) a aplicaçāo de um código tipo CSOC ("Convolutional SelfOrthogonal Code") é de especial interesse, uma vez que o correspondente algoritmo permite o uso de decisāo suave e implica num custo reduzido de hardware [10] e [11]. Caso o sistema permita mais redundância, códigos do tipo PCC ("Punctured Convolutional Codes") [12] e decodificaçāo tipo Viterbi podem ser levados em consideração.

Na Fig. 4 estāo plotados os resultados de alguns cálculos e simulaçōes, supondo-se o canal AWGN ("Additive White Gaussian Noise"), na qual sāo comparadas as taxas de erro de bit (TEB) dos seguintes esquemas:

(i) 256-QAM sem codificaçāo;

(ii) 256-QAM utilizando CSOC com taxa de codificação $R_{c}=8 / 9$ e distância $\mathrm{d}=5$, decodificado por um algoritmo decodificador de limiar simplificado;

(iii) 512-TCM de Ungerboeck utilizando a taxa $R_{c}=2 / 3$ e um código convolucional de 16 estados e decodificaçāo por Viterbi. 


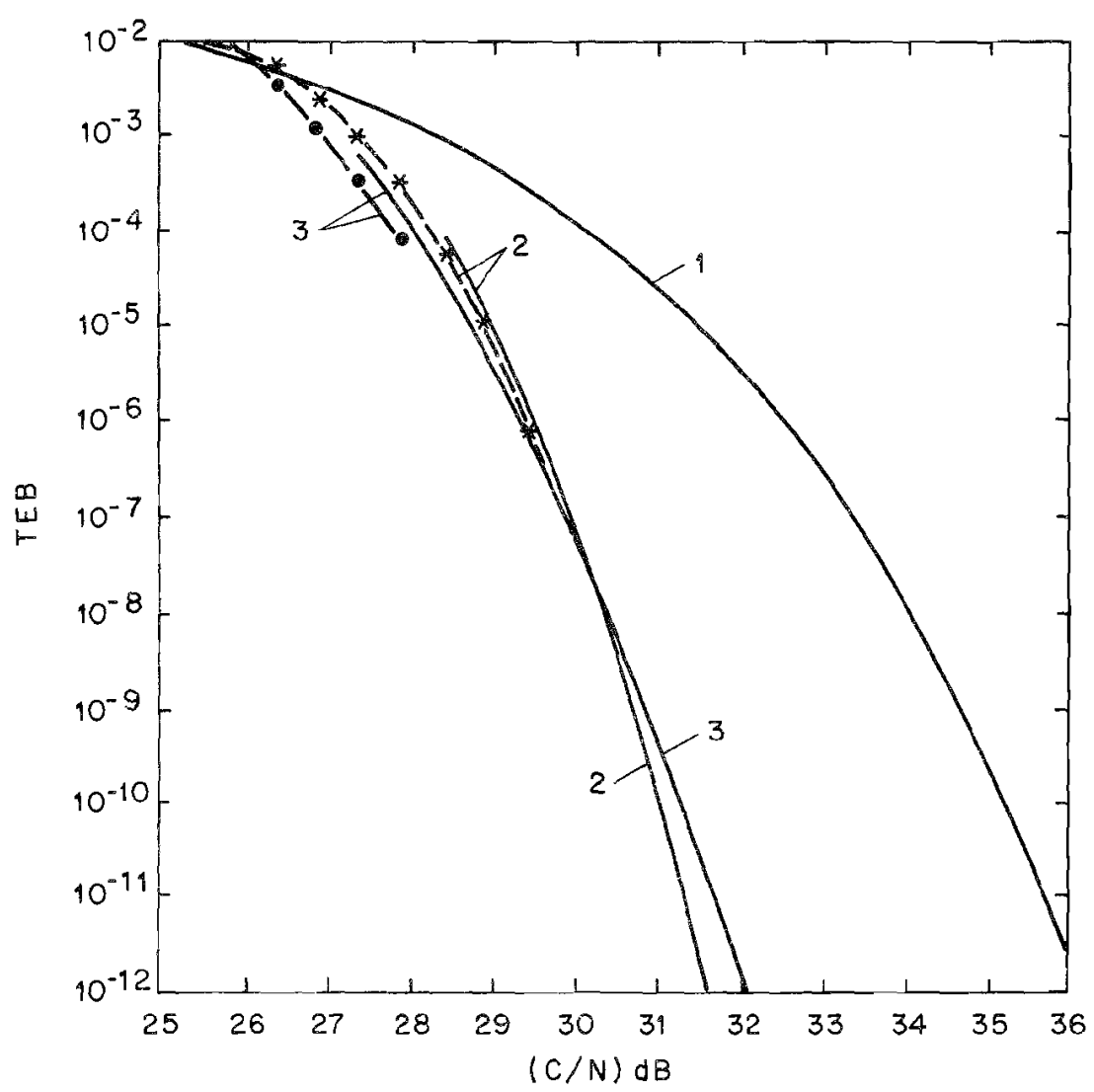

Figura 4. Resultados de cálculo e de simulaçāo para taxa de erro de bit (TEB) versus razão portadora-ruído (C/N) : (1) 256-QAM sem codificação;

(2) 256-UC-QAM, CSOC, $R_{C}=8 / 9, R_{g}=35 / 36$; (3) 512-TCM, $R_{C}=2 / 3$, 16 estados ( ${ }^{*}, \because$ Simulaçāo; Linha cheia: Cálculo).

Os símbolos (*) e (•) na Fig. 4 indicam resultados de simulaçōes enquanto as linhas cheias resultam de cálculos de acordo com [4]. Considerando os ganhos assintóticos, pode-se ver que dispondo apenas de uma redundância global menor que 3\%, o UC-QAM é superior ao TCM. Um decodificador CSOC com taxa de $8 / 9$ e com distância $d=5$ pode ser implementado num circuito integrado "semicustom" (tecnologia 1,5 $\mu$ ) necessitando menos que 10.000 portas equivalentes. Dessa maneira, o custo de hardware para a implementação do 256-UC-QAM com taxa 8/9 e CSOC é consideravelmente menor do que para o 512-TCM com decodificaçāo de Viterbi de 16 estados. 
Para taxas de erro elevadas O TCM é ligeiramente melhor que o UC-QAM usando CSOC. Isso se deve ao fato de que o algoritmo de decodificaçāo de limiar nāo atinge a propriedade de "Maximum Likelihood Detection" enquanto o algoritmo de Viterbi o faz.

\section{Conclusões}

Foi apresentada uma técnica de codificaçāo com redundância eficiente para sistemas rádio digitais com modulação QAM de grau elevado. Com essa técnica atinge-se ganhos de codificaçāo de até $6 \mathrm{~dB}$, necessitando-se para isso, apenas um pequeno aumento na largura de faixa. As tecnologias atualmente disponiveis permitem uma implementação a custo moderado.

\section{Referências}

[1] T. Noguchi, Y. Daido e J.A. Nossek, "Modulation Techniques for Microwave Digital Radio", IEEE Communications Magazine, vol. 24, $n^{\circ}$ 10, Outubro 1986, pp. 21-30.

[2] R. Steinhart e F. Ziss, "Neuere Entwicklunstendenzen und Markttendenzen in der Richtfunktechinik", NTZ, vol. 41, Novembro 1988, pp. 618-621.

[3] J.A. Nossek Steinkamp, "Digitalrichtfunk, ein Technologieorientiertes wirtschaftliches-Uebertragungsmedium", NTZ, vol. 41, Novembro 1988, pp. 622-629.

[4] G.C. Clark, Jr. e J.B. Cain, "Error-Correcting Codes for Digital Communications", New York, Plenum Press, 1982.

[5] G. Ungerboeck, "Channel Coding with Multilevel/Phase Signals", IEEE Transactions on Information Theory, vol. IT-28, $n^{0} 1$, Janeiro 1982, pp. 55-67.

[6] G. Ungerboeck, "Trellis-Coded Modulation with Redundant Signal Sets, Parts I and II", IEEE Communications Magazine, vol. 25, n², Fevereiro 1987, pp. 5-21.

[7] J. Hagenauer e C-E. Sundberg, "On the Performance Evaluation of Trellis-Coded 8-PSK Systems with Carrier Phase Offset", AEU, vol. 42, Outubro 1988, pp. 274-284. 
[8] P. Mecklenburg, W.K. Pehlert, Jr. e D.D. Sullivan, "Correction of Errors in Multilevel Gray-Coded Data", IEEE Transactions on Information Theory, vol. IT-19, $n^{9}$ 3, Maio 1973, pp. 336-340.

[9] A.J. Viterhi e J.K. Omura, "Principles of Digital Communications and Coding", New York, McGraw Hill, 1979.

[10] J.L. Massey, "Threshold Decoding", Cambridge, Massachusetts, MIT Press, 1963.

[11] H. Tanaka, K. Furusawa e S. Kanebu, "A Novel Approach to Soft Decision Decoding of Threshold Decodable Codes", IEEE Transactions on Information Theory, vol. IT-26, $n^{\circ} 2$, Março 1980, pp. 244-246.

[12] Y. Yasuda, K Kachiki e Y. Hirata, "High-Rate Punctured Codes for Soft Decision Viterbi Decoding", IEEE Transactions on Communications, vol. COM-32, $n^{9}$ 3, Março 1984, pp. 315-319.

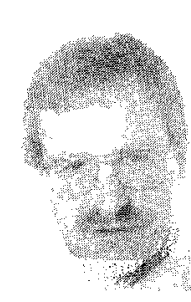

KARL - JOSEF FRIEDERICHS nasceu em 1955. Ele recebeu o grau Ing. Grad. da Fachhochschule Aachen, Alemanha, em 1979 e os graus Dipl. Ing. e PhD da Universidade de Kaiserslautern, Alemanha, em 1982 e 1986, respectivamente. Desde 1987, ele está com o Departamento de Projeto de Sistemas Rádio da Siemens AG, Munich, onde chefia atualmente o grupo de desenvolvimento. Seus interesses de pesquisa incluem codificação, detecção, sincronização e técnicas rápidas de processamento digital de sinais para sistemas de comunicações. Dr. Friederichs é membro da IEEE Communications Society e da Informationstechnische Gesselschaft (ITG).

RONALD MARTIN DAUSCHA nasceu no Rio de Janeiro, em 14 de outubro de 1961. É formado em Engenharia Eletrônica (especialização Telecomunicações) pela Escola Politécnica da Universidade de São Paulo. Desde 1984, atua na área de desenvolvimento de equipamentos de transmissão da Equitel S.A. De 1986 a 1989 participou do desenvolvimento de sistemas rádio digital de alta capacidade nos laboratórios da Siemens, Munich. 